\title{
Development of the Measurement Method for Challenging NLOS Conditions in Mobile Networks
}

\author{
Marjo Heikkilä, Juha Erkkilä, \\ Marjut Koskela, Joni Heikkilä, \\ Tuomo Kupiainen \\ Centria University of Applied \\ Sciences \\ Ylivieska, Finland
}

\author{
Jouni Tervonen \\ University of Oulu \\ Nivala, Finland
}

\author{
Marco Migliore \\ University of Cassino and Southern \\ Lazio \\ Cassino, Italy
}

\begin{abstract}
The aim of this paper is to introduce the measurement method for challenging Non-Line of Sight (NLOS) conditions in mobile networks. The need to develop the measurement method appeared LTE (Long Term Evolution) uplink (UL) performance with different antenna technologies when receiving NLOS signal in field tests. Many challenges appeared during the study. This paper introduces the challenges and presents solution.
\end{abstract}

Keywords-Vertical beamforming; Horizontal beamforming; AAS; Non-line of Sight Environment; Uplink capacity improvement; 2and 4-way $R X$ diversity; Field trial; MIMO

\section{INTRODUCTION}

The world is moving forward to the digital age. The $3 \mathrm{G}$ and $4 \mathrm{G}$ have brought increasing amounts of digital information available to people. With mobile networks, files can be shared, movies can be watched and updates to social media can be given with smart phones around the clock. The machine-to-machine (M2M) type communication is accelerating and it will effect everyday life, industries and cities, even critical infrastructure communication. Increasingly more intelligence is built into devices. These includes sensors, location awareness, communication and processing abilities. The $5 \mathrm{G}$ will bring capabilities that take digitalization to the new level. It will enable M2M communication with low latencies. It will also deliver speeds up to $10 \mathrm{Gbit} / \mathrm{s}$. This will enable high-speed broadband service for the critical type of machine communication demanding low latency and high bandwidth.

The demands and features of cellular communication are accumulating. The future network needs to be more dynamic to handle different types of communications. It is essential for networks to adapt according to varying needs. There are several features that can make networks more flexible like dynamic spectrum management and beamforming. When the complexity of networks increases, the verification of the network performance becomes more and more challenging. The demand for high reliability and availability of the network must be verified with testing.

This paper illustrates the practical challenges of measuring dynamic cellular networks. This study focuses on developing the field test method for Active Antenna Systems (AAS). AAS with $3 \mathrm{D}$ beamforming feature is a viable solution increasing the capacity of the network. In the 3D beamforming, the beam can be formed horizontally and vertically. In addition to directing the beam, tilt and azimuth can also be changed. With all these adjustments, network throughput can be improved [1]. This study expands the previous work on AAS field trial [2].

The Section I illustrates aspects that affect mobile networks. The factors affecting the measurement in mobile networks are described in Section II. The measurement setup and environment are described in Section III. Selection of the measurement points is explained in Section IV. The description of measurement case is shown in Section V and the results are shown in Section VI. Discussion and conclusion are shown in Section VII.

\section{FACTORS AFFECTING THE MEASUREMENT IN MOBILE NETWORKS}

Several factors must be considered when the verification measurements of the mobile network are planned. The mobile network and system parameters need specific attention when measurements are planned. Environmental factors significantly impact on the signal propagation. When measurements are planned, they often demand additional definition afterwards

\section{A. LTE technology}

The LTE capacity depends on many issues such as the data transmission capability of single cells. The mobile network and parameters need to be designed appropriately to get optimal coverage and capacity. Network parameters such as radiated power, frequency band, bandwidth, antenna design, and power need to be considered. The capacity is close to the number of the LTE evolved Node B (eNB) elements and to the bandwidth each eNB is offering. The distance between eNB and User Equipment (UE) also affects the capacity. The dynamic modulation and coding scheme results in high data rates and capacity when the UE is located near the eNB, whereas the cell edge and inside building locations offer minimum capacity. As the bit error rate increases along with the utilization level of the network it also influences the signal coverage areas of the eNBs [3] 


\section{B. $M I M O$}

Multiple-Input Multiple-Output (MIMO) is a radio communications technology that simultaneously utilizes multiple spatially distributed antennas. These multiple antennas act as transmitters (TX) and receivers (RX), which enable a variety of different signal paths for each antenna see Figure 1. This enables multiple signal paths to be utilized to transmit the data. MIMO antennas can use spatial diversity and spatial multiplexing formats in data transmit. Spatial diversity improves the signal to noise ratio and thus improves the reliability. Spatial multiplexing provides increase in data throughput by utilizing the different paths to transmit the data traffic.

\section{Environmental impacts on signal propagation}

Environment affects signals in many ways depending on the surroundings. In the line of sight (LOS) signal propagation, there are no obstacles between UE and transmitter antenna. Multipath causes the largest effect, which can be destructive or constructive. Obstacles such as buildings and vehicles around UE produce reflections and multipath propagation. Each path has a specific delay, attenuation and phase shift feature. The signal attenuates on the way from the transmitted antenna to the receiver because the signal energy spreads around the transmitter. The UE receives and sums up multiple copies of the signal with different phases and amplitudes. Arriving signals can be out of the phase and may gain or attenuate each other. Buildings will cause losses that are dependent on the electrical properties of the materials [4].

There are practical studies of energy-efficient construction practices that have effect on RF signals by increasing entry losses [5]. The roughness of surfaces fluctuates the power of scattering waves. The power of scattering waves depends on the frequency of the incident wave [4].

The environmental effect of trees is quite widely studied. There are many studies e.g., [6], [7] and [8], which have reported the seasonal effect i.e., the increase in the attenuation of trees inleaf compared with the out-of-leaf state.

\section{Weather impact on the signal propagation}

Effects of the weather on the signal propagation are mainly related to the attenuation by atmospheric gasses and rain.

Oxygen and water vapor in the atmosphere cause strong absorption at resonance frequencies. However, such frequencies are above $10 \mathrm{GHz}$. As a consequence atmospheric gasses absorption at frequency below $10 \mathrm{GHz}$ is lower than 0.01 $\mathrm{dB} / \mathrm{Km}$ and its effect can be ignored [9].

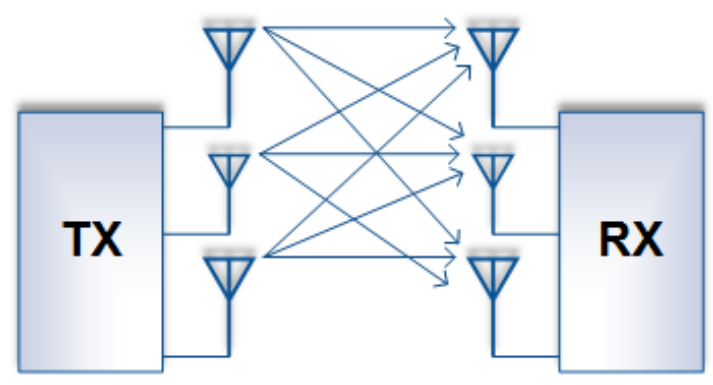

Fig. 1. Basic MIMO concept.
The rain affects radio wave propagation in many ways. The main effect is the attenuation of the radio signal caused by the absorption of power by water droplets. Furthermore, there is a loss of power of the signal between transmitter and receiver due to the scattering of the water droplets.

Water droplet diameter ranges from fractions of millimeters in case of light rain to some millimeters in case of heavy rain. Consequently, it is possible to use the Rayleigh model to evaluate absorption and scattering of droplets up to several $\mathrm{GHz}$ [10].

The studies carried out showed that the attenuation is lower than $0.1 \mathrm{~dB} / \mathrm{km}$ frequency up to $10 \mathrm{GHz}$ in case of moderate rain $(5 \mathrm{~mm} / \mathrm{h})$, while at $5 \mathrm{GHz}$ the attenuation is lower than 0.1 $\mathrm{dB} / \mathrm{km}$ also in case of heavier rain $(20 \mathrm{~mm} / \mathrm{h})$. Regarding the scattering, its effect is small compared to the one related to the power absorption. Depolarization effect is also small [11]. Consequently at ultra-high frequencies (UHF) rain does not significantly affect the signal propagation [12].

Water is also present in fog. However droplets in fog have radius of the order of $1 / 100^{\text {th }}$ of $\mathrm{mm}$, giving a negligible absorption in UHF.

Finally, it is worth noting that rain can affect the signal also in a 'indirect way', changing the electromagnetic environment. In fact, wet surfaces have reflective properties different from dry surfaces. Since the electromagnetic environment depends on the reflecting properties of the surfaces, wet surfaces modify the multipath propagation and hence the communication channel between transmitter and receiver.

\section{MEASUREMENT ENVIRONMENT AND SETUP}

The purpose of original measurements was to study the effect of UL beamforming to reduce extreme path loss with NLOS signals. The aim of the measurements was to compare UL performance in the challenging radio environment with different antenna technologies. The measurements were performed in a suburban/rural environment within the field trial environment which is located in Ylivieska, Finland [13]. Comparison was made between the passive antenna, horizontal AAS and vertical AAS in case of NLOS signal. The conclusion of the results was that there is not only one configuration feature that gives the best throughput from every measurement location. The highest throughput gain was provided with vertical AAS, but the best configuration for the single measurement point was varying from point to point. The use of different elevation beams and RX diversity gives the most advanced signal levels in NLOS environment. The vertical AAS was the most promising in these tests. When the beam is steered towards the user, the horizontal beam forming is the most useful [2].

\section{A. Field trial environment}

The field trial environment of Centria University of Applied Sciences covers approximately $4 \mathrm{~km}^{2}$ area in rural/suburban environment of Ylivieska. Puuhkala eNB was chosen for these measurements, for the Puuhkala site has the most versatile antenna techniques available. Puuhkala eNB is a MIMO AAS while utilizing a UL Coordinated Multipoint (CoMP) feature. 


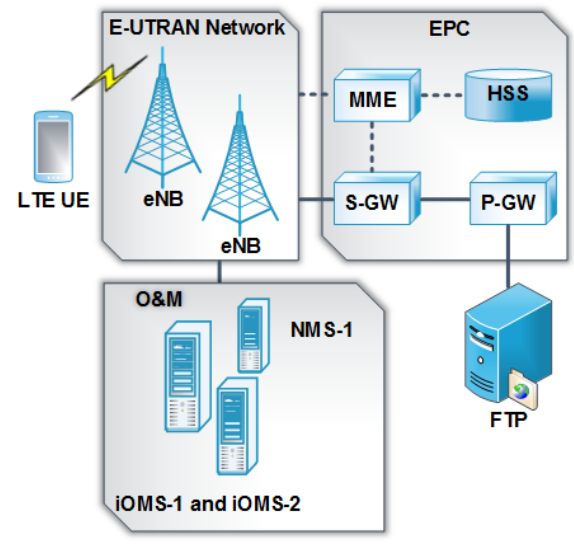

Fig. 2. Field trial network.

UL CoMP is a complex technique which brings many advantages to the network. These advantages are better the utilization of the network, interference reduction, enhanced reception performance and received power increase in multisite reception. Measurements were conducted in a field trial network, which has a restricted connection to the internet or other public networks. This network can be operated only by UEs which are acquired for test purpose see Figure 2. The Evolved Universal Mobile Telecommunications System Terrestrial Radio Access Network (E-UTRAN) is the access network, which consists of LTE radio technique eNBs. The eNBs are connected to Evolved Packet Core (EPC) and to Operation and Maintenance (O\&M). In the measurement configuration, two AAS and one passive antenna in $2.1 \mathrm{GHz}$ LTE band at $5 \mathrm{MHz}$ LTE system bandwidth were operated from Puuhkala eNB [2].

\section{B. Measurement setup}

All performance evaluation measurements were performed with same measurement devices, including LTE dongle [14], external antennas [15], laptop and measurement software [16]. The LTE dongle utilized a Multiple Input Single Output (MISO) internal antenna technique. Different measurement setups were used for indoor and outdoor test cases. During the indoor measurements, the test setup was moved inside the buildings. In the indoor measurements, external antennas were not connected with the LTE dongle and only internal antennas of the LTE dongle were used. During the outdoor measurements, a car was equipped with the full test setup. The LTE dongle was equipped with external antennas, a mere RX antenna located in the middle of instrument panel, TX/ RX antenna located on the roof of the car. A laptop with the measurement software was located on the front seat of the car.

\section{Measurement period}

The conducted measurements took place from early June to late September. During the four-month measurement period, only considerable changes in the measurement circumstances were that the environment foliage decreases in autumn, which caused a slight throughput increase in the measurements [2]. This effect is expected as many studies have reported the decrease of the attenuation of trees without foliage e.g. [6], [7] and [8]. The weather was visually observed during the measurements and it was established not include in weather effects on the results.

\section{SELECTION OF THE MEASUREMENT POINTS}

At the beginning of this study, it was important to find the most suitable measurement points with challenging locations that are able to give meaningful information on the performance of complex communication system. The procedure started with defining the specification for challenging points between the $\mathrm{UE}$ and the eNB. The objective was to find locations with a challenging radio environment. The challenging points were chosen so that the environment had Non-Line of Sight (NLOS) signal propagation to the Puuhkala eNB. Eleven different measurement points were selected for the preliminary study according to assumptions of challenging environment based on the aspect such as surrounding buildings, distance and direction from antenna mast as well as the estimation of the coverage area. The preliminary measurements gave more accurate information to plan the actual comparison measurements.

\section{A. Preliminary measurements}

Puuhkala eNB (brown point: eNB) and measurement points are shown in Figure 3. Three of the eleven measurement points were located indoors (blue points: MP 9, MP 10, MP 11). The remaining eight measurement points were located outdoors (red points: from MP 1 to MP 8). Common to the outdoor measurement points was that the material used in the surrounding buildings is mainly brick and concrete element.

Two of the three indoor measurement points were located in the proximity of the Puuhkala eNB and MP 11 was located inside Centria's campus. After the first preliminary measurements, it was concluded from the analysis that indoor measurement points MP 9, MP 10 and MP 11 did not meet the specific requirements for these measurements, because these measurement points gave exceptionally good UL throughput with every antenna configuration even while the measurement points were located inside concrete buildings. It was concluded that the good UL throughput was because these indoor measurement points were located near the Puuhkala eNB. The

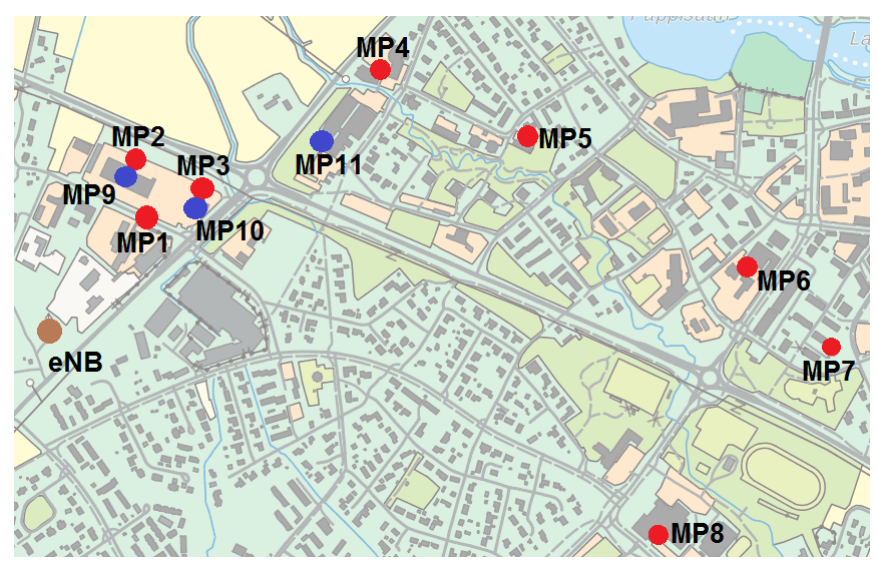

Fig. 3. Location of measurement points and Puuhkala eNB in Ylivieska. 
signal strength as well as signal-to-noise ratio was good and enabled the best possible UL throughput with these setups.

Outdoor measurement points MP 1, MP 2 and MP 3 were too close to the eNB and gave the best possible UL throughput in the preliminary measurements. In the preliminary analysis, it was concluded that these measurement points did not meet the requirements and did not have a sufficiently challenging environment that was set in the specification for these measurements.

\section{B. Accepted measurement points in detail}

Based on the analysis from the preliminary measurements, it was concluded that five measurement points; MP 4, MP 5, MP 6, MP 7 and MP 8 would be suitable for actual measurements. Most of these measurement points have high buildings obscuring the LOS from Puuhkala eNB and the measurement points have neighboring buildings near them. The height difference between Puuhkala eNB antenna element and measurement point MP 4 to MP 8 has been calculated in Table I. The height values of eNB and MP's are based on GPS information. The distance from the measurement points to Puuhkala eNB and height of the buildings obscuring the LOS at each measurement point is shown in Table I.

The building obscuring the LOS at the measurement point MP 4 between the eNB and UE is four-storey high office building whose outer wall is made of bricks. Behind this office building is a one-storey high office building with very large cone-shaped roof. The yard has been coated with asphalt and serves as a parking lot for the workers.

At the measurement point MP 5, the building between the eNB and the measurement point is a three-storey high apartment building whose outer wall is made of bricks. The yard of this building has been coated with asphalt. Behind this measurement point is an apartment building, whose outer wall is also made of bricks. Near these buildings grow a number of birch and pine trees.

The building between the measurement point MP 6 and eNB is a one-storey high community building whose outer wall is made of wood. This measurement point is located in the courtyard of the community building and a four-storey high office building. The office building and other buildings around this measurement point have outer walls made of bricks and the courtyard is coated with asphalt. This courtyard has parking places for the worker's cars and the area was almost full of them during the measurement.

The building between measurement point MP 7 and the eNB is a four-storey high apartment building whose outer wall is

TABLE I. MEASUREMENT POINT INFORMATION

\begin{tabular}{|c|c|c|c|}
\hline $\begin{array}{c}\text { Measurement } \\
\text { points (MP) }\end{array}$ & $\begin{array}{c}\text { Distance to } \\
\text { Puuhkala } \\
\text { eNB }\end{array}$ & $\begin{array}{c}\text { Height of } \\
\text { building } \\
\text { obscuring } \\
\text { LOS }\end{array}$ & $\begin{array}{c}\text { Height difference } \\
\text { between eNB } \\
\text { antenna and the } \\
\text { MP }\end{array}$ \\
\hline MP 4 & $830 \mathrm{~m}$ & $18.5 \mathrm{~m}$ & 57 \\
\hline MP 5 & $1014 \mathrm{~m}$ & $14.5 \mathrm{~m}$ & 60 \\
\hline MP 6 & $1380 \mathrm{~m}$ & $11.0 \mathrm{~m}$ & 37 \\
\hline MP 7 & $1547 \mathrm{~m}$ & $12.0 \mathrm{~m}$ & 56 \\
\hline MP 8 & $1271 \mathrm{~m}$ & $10.0 \mathrm{~m}$ & 47 \\
\hline
\end{tabular}

made of bricks. Near this building grow a number of birch trees. In the proximity of MP 7 is an asphalt parking space for the residents.

Measurement point MP 8 has a tennis hall with arched roof between the UE and eNB. In the immediate vicinity of the tennis hall are no other tall buildings. A few hundred meters from the tennis hall is an indoor ice rink. The asphalt coated inner yard between the tennis hall and the indoor ice rink serves as a parking space.

\section{DESCRIPTION OF MEASUREMENT CASE}

In the chosen measurement points from MP 4 to MP 8, repeatability, reliability and good results in challenging radio environment were investigated in the measurements. At each measurement point, the measurement began by placing the UE in a predetermined location, which was evaluated visually, and the UL data transfer could be started.

One measurement lasted approximately one minute, during which time the drive test software gathered approximately two samples per second. During one measurement, on average the amount of throughput samples gathered was $150 \pm 20$.

The measurement software [16] recorded media access control (MAC) throughput, reference signal received power (RSRP), transmit (TX) power, reference signal received quality (RSRQ) and signal to noise ratio (SNR) from measurements. Location was identified by Global Positioning System (GPS) and this information was utilized in the analyzing phase. Data transfer was conducted by File Transfer Protocol (FTP). The drive test software recorded the FTP data transfer using automated scripts. In the analysis phase, it was discovered that the measurement software recorded some sample values before and after the data transfer, which were not part of the actual data transfer. These false values were filtered from the results in the analysis phase [2].

\section{A. Stationary measurements}

UE (the car) was stationary during the first phase of measurements. Measurement analysis indicated that the static UE measurements in measurement points had too much variation and results were not repeatable. This is due to signal reflections on the environment and the difficulty to place the UE again in exactly the same spot for every measurement. It was concluded that measurement procedure had to be developed in order to obtain statistical and reliable measurement results.

\section{B. Moving measurements}

The assumption was that the results varied because of challenging radio environment. The study was performed in NLOS locations where the reflections of the transmitted signal could change because of environment.

The aim was to eliminate the effect of changes in signal reflections. The measurements were repeated with moving UE. The car with the measurement equipment was driven very slowly parallel to NLOS point buildings. The measurements were repeated forwards and backwards to see if the moving direction had an effect on the results. It appeared that the results 
were more reliable and repeatable when the UE was moving slowly.

\section{RESULTS}

We performed several moving and stationary measurements with different antenna configurations. In this Section, we analyze the distributions of measurements on different dates or times. When the measurement is reliable and repeatable, the distributions of different measurements should not differ much. In the first analysis, we have compared the distributions using the mean and the standard deviations, which are the main parameters of the distributions and normal i.e., Gaussian distributions are commonly statistically compared using these parameters. We have adapted this kind of comparison without the normality check on the compared distributions to show some results across the whole measurement data. Table II gives the variances of the mean $(\mu)$ and the standard deviations $(\sigma)$ with different antenna configurations and different measurement points (MP) with stationary (Stat) or moving (Mov) measurements. As shown in Table II, the number of measurements $(\mathrm{N})$ per method and measurement sites varied from three to nine measured distributions. Table II reveals that the deviations between the mean values of measurements are in all cases smaller with moving measurements. The similar behavior with few exceptions is also found with the standard deviations. These results suggest that moving measurements are more repeatable.

Since the difference between moving and stationary measurements is particularly high at measurement point MP 5 at 4-Way RX AAS method this site/method is further studied. A comparison in a form of descriptive statistics, the boxplot visualizations of the different distributions are given in Figure 4. This clearly shows more considerable variations of the means and medians of stationary measurement compared with the moving measurements. Also the dispersion is higher at

TABLE II. DEVIATIONS OF MEASURED DISTRIBUTIONS

\begin{tabular}{|c|l|c|c|c|}
\hline \multicolumn{2}{|c|}{ Methods/sites } & $\begin{array}{c}\text { 2-Way RX } \\
\text { passive }\end{array}$ & $\begin{array}{c}\text { 4-Way RX } \\
\text { passive }\end{array}$ & $\begin{array}{c}\text { 4-Way RX } \\
\text { AAS }\end{array}$ \\
\hline \multirow{4}{*}{ MP 4 } & Var $\mu$ Stat & $\mathrm{NA}^{\mathrm{a}}$ & $0.065(\mathrm{~N}=3)$ & $0.110(\mathrm{~N}=4)$ \\
\cline { 2 - 5 } & Var $\mu$ Mov & $0.008(\mathrm{~N}=5)$ & $0.013(\mathrm{~N}=5)$ & $0.053(\mathrm{~N}=5)$ \\
\cline { 2 - 5 } & Var $\sigma$ Stat & $\mathrm{NA}$ & $0.036(\mathrm{~N}=3)$ & $0.007(\mathrm{~N}=4)$ \\
\cline { 2 - 5 } & Var $\sigma$ Mov & $0.008(\mathrm{~N}=5)$ & $0.013(\mathrm{~N}=5)$ & $0.005(\mathrm{~N}=5)$ \\
\hline \multirow{4}{*}{ MP 5 } & Var $\mu$ Stat & $0.059(\mathrm{~N}=5)$ & $0.489(\mathrm{~N}=7)$ & $1.305(\mathrm{~N}=8)$ \\
\cline { 2 - 5 } & Var $\mu$ Mov & $0.045(\mathrm{~N}=9)$ & $0.034(\mathrm{~N}=9)$ & $0.043(\mathrm{~N}=9)$ \\
\cline { 2 - 5 } & Var $\sigma$ Stat & $0.006(\mathrm{~N}=5)$ & $0.010(\mathrm{~N}=7)$ & $0.117(\mathrm{~N}=8)$ \\
\cline { 2 - 5 } & Var $\sigma$ Mov & $0.007(\mathrm{~N}=9)$ & $0.001(\mathrm{~N}=9)$ & $0.010(\mathrm{~N}=9)$ \\
\hline \multirow{4}{*}{ MP 6 6} & Var $\mu$ Stat & $0.043(\mathrm{~N}=3)$ & $0.395(\mathrm{~N}=7)$ & $2.007(\mathrm{~N}=8)$ \\
\cline { 2 - 5 } & Var $\mu$ Mov & $0.032(\mathrm{~N}=9)$ & $0.031(\mathrm{~N}=9)$ & $0.140(\mathrm{~N}=9)$ \\
\cline { 2 - 5 } & Var $\sigma$ Stat & $0.006(\mathrm{~N}=3)$ & $0.003(\mathrm{~N}=7)$ & $0.115(\mathrm{~N}=8)$ \\
\cline { 2 - 5 } & Var $\sigma$ Mov & $0.004(\mathrm{~N}=9)$ & $0.021(\mathrm{~N}=9)$ & $0.023(\mathrm{~N}=9)$ \\
\hline \multirow{4}{*}{ MP 7 } & Var $\mu$ Stat & $\mathrm{NA}$ & $0.394(\mathrm{~N}=2)$ & $0.722(\mathrm{~N}=3)$ \\
\cline { 2 - 5 } & Var $\mu$ Mov & $0.060(\mathrm{~N}=5)$ & $0.125(\mathrm{~N}=5)$ & $0.071(\mathrm{~N}=5)$ \\
\cline { 2 - 5 } & Var $\sigma$ Stat & $\mathrm{NA}$ & $0.001(\mathrm{~N}=2)$ & $0.035(\mathrm{~N}=3)$ \\
\cline { 2 - 5 } & Var $\sigma$ Mov & $0.008(\mathrm{~N}=5)$ & $0.032(\mathrm{~N}=5)$ & $0.066(\mathrm{~N}=5)$ \\
\hline \multirow{4}{*}{ MP 8 } & Var $\mu$ Stat & $\mathrm{NA}$ & $0.156(\mathrm{~N}=2)$ & $1.340(\mathrm{~N}=3)$ \\
\cline { 2 - 5 } & Var $\mu$ Mov & $0.018(\mathrm{~N}=5)$ & $0.042(\mathrm{~N}=5)$ & $0.030(\mathrm{~N}=5)$ \\
\cline { 2 - 5 } & Var $\sigma$ Stat & $\mathrm{NA}$ & $0.036(\mathrm{~N}=2)$ & $0.074(\mathrm{~N}=3)$ \\
\cline { 2 - 5 } & Var $\sigma$ Mov & $0.005(\mathrm{~N}=5)$ & $0.016(\mathrm{~N}=5)$ & $0.010(\mathrm{~N}=5)$ \\
\hline \multirow{4}{|c|}{} & & & \\
& \multicolumn{4}{|c}{ Not available (NA) }
\end{tabular}

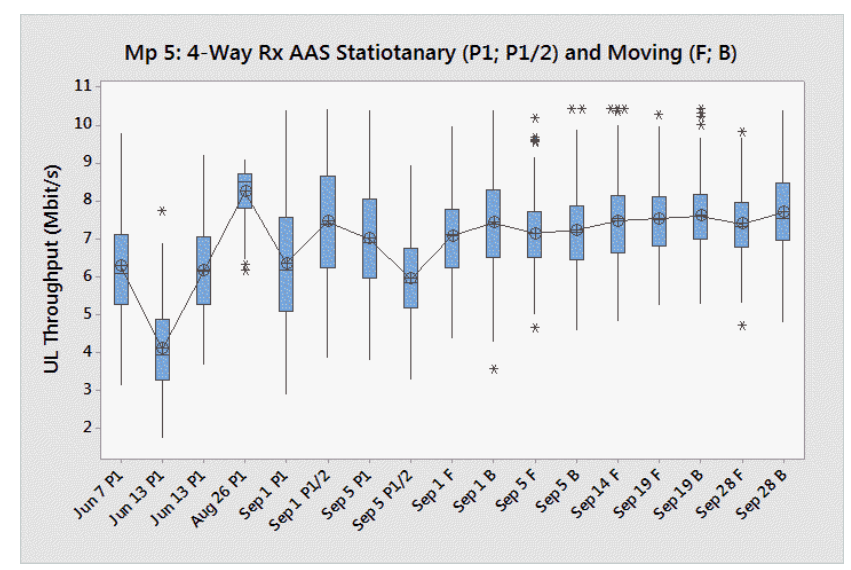

Fig. 4. Measured 4-Way AAS distribution at MP 5.

stationary measurements. The smaller dispersion at moving measurement is a positive wanted indication of the more repeatable measurement method, but as a side effect the smaller dispersion will also cause that a few more extreme will values are interpreted as outliers in the boxplot analysis. The smaller box, i.e., difference between and the first quartile, affect the smaller maximum length of the whiskers meaning tighter criterion for outliers.

In the last statistical analysis, the similarities of the measurement distributions are pair-wise checked with twosample Kolmogorov-Smirnov test (kstest). The test returns a decision for the null hypothesis that data in the compared two sample distributions come from the same continuous distribution. The result of kstest is 1 if the test rejects the null hypothesis at the selected significance level, and 0 otherwise. We performed the pair-wise tests with Matlab at the $1 \%$ significance level. The results of pair-wise kstests for stationary and moving measurements are given in Tables III and IV, respectively. In the moving measurements, $75.0 \%$ of the nondiagonal different measurements lead to the same distribution while the rate is only $17.9 \%$ at stationary measurements.

These analyses clearly reveal the outperformance of the moving measurement method in order to produce reliable and repetitive results.

Results of the moving measurement were more reliable and repeatable because in motion the most extreme and deep fadings due to reflections or other properties of the signal connection between the eNB and UE were averaged out. The more there are reflections the better is the connection, because the used technique - LTE Single Input Multiple Output (SIMO) benefits from the fact that considerable amount of reflections appear as long as they are received by the UE sufficiently at different times. Moving forward or backward has no significant difference. This was noticed in the analysis of the measurement results.

\section{DISCUSSION AND CONCLUSION}

This article describes test method development in field trials when uplink throughput gain was evaluated. Trials were performed in the non-line of sight environment while using 
TABLE III. TWO-SAMPLE KOLMOGOROV-SMIRNOV TEST OF STATIONARY MEASUREMENTS

\begin{tabular}{|c|c|c|c|c|c|c|c|c|}
\hline $\begin{array}{c}\text { 4-Way } \\
\text { RX AAS } \\
\text { at 0.01 }\end{array}$ & Jun-07 & \multicolumn{2}{|c|}{ Jun-13 } & Aug-26 & \multicolumn{2}{|c|}{ Sep-01 } & \multicolumn{2}{|c|}{ Sep-05 } \\
\cline { 2 - 9 } & $\boldsymbol{S 1}$ & $\boldsymbol{S 2}$ & $\boldsymbol{S 3}$ & $\boldsymbol{S 4}$ & $\boldsymbol{S 5}$ & $\boldsymbol{S 6}$ & $\boldsymbol{S} 7$ & $\boldsymbol{S} 8$ \\
\hline S1 & 0 & 1 & 0 & 1 & 0 & 1 & 1 & 0 \\
\hline S2 & 1 & 0 & 1 & 1 & 1 & 1 & 1 & 1 \\
\hline S3 & 0 & 1 & 0 & 1 & 0 & 1 & 1 & 0 \\
\hline S4 & 1 & 1 & 1 & 0 & 1 & 1 & 1 & 1 \\
\hline S5 & 0 & 1 & 0 & 1 & 0 & 1 & 1 & 1 \\
\hline S6 & 1 & 1 & 1 & 1 & 1 & 0 & 1 & 1 \\
\hline S7 & 1 & 1 & 1 & 1 & 1 & 1 & 0 & 1 \\
\hline S8 & 0 & 1 & 0 & 1 & 1 & 1 & 1 & 0 \\
\hline
\end{tabular}

TABLE IV. TWO-SAMPLE KOLMOGOROV-SMIRNOV TEST OF MOVING MEASUREMENTS

\begin{tabular}{|c|c|c|c|c|c|c|c|c|c|}
\hline \multirow{2}{*}{$\begin{array}{c}\text { 4-Way } \\
\text { RX AAS } \\
\text { at 0.01 }\end{array}$} & \multicolumn{2}{|c|}{ Sep-01 } & \multicolumn{2}{c|}{ Sep-05 } & Sep-14 & \multicolumn{2}{c|}{ Sep-19 } & \multicolumn{2}{c|}{ Sep-28 } \\
\cline { 2 - 11 } & M1 & M2 & M3 & M4 & M5 & M6 & M7 & M8 & M9 \\
\hline M1 & 0 & 0 & 0 & 0 & 1 & 1 & 1 & 1 & 1 \\
\hline M2 & 0 & 0 & 1 & 0 & 0 & 0 & 0 & 0 & 0 \\
\hline M3 & 0 & 1 & 0 & 0 & 0 & 1 & 1 & 0 & 1 \\
\hline M4 & 0 & 0 & 0 & 0 & 0 & 0 & 1 & 0 & 1 \\
\hline M5 & 1 & 0 & 0 & 0 & 0 & 0 & 0 & 0 & 0 \\
\hline M6 & 1 & 0 & 1 & 0 & 0 & 0 & 0 & 0 & 0 \\
\hline M7 & 1 & 0 & 1 & 1 & 0 & 0 & 0 & 0 & 0 \\
\hline M8 & 1 & 0 & 0 & 0 & 0 & 0 & 0 & 0 & 0 \\
\hline M9 & 1 & 0 & 1 & 1 & 0 & 0 & 0 & 0 & 0 \\
\hline
\end{tabular}

passive, 2-way and 4-way RX diversity in horizontal and vertical beamforming in suburban/rural area of Ylivieska, Finland. The study with statistical analyses clearly revealed that measurements in challenging radio propagation environment with moving UE give more reliable and repeatable results than measurements with stationary located UE.

Changes in the next generation mobile networks cause need to further develop into field test methods. There is a need to develop the test methods in order to evaluate the performance of the future dynamic mobile networks. The potential of unmanned aircraft systems will be researched in the future trials to meet challenges of testing the mobile network with 3D beamforming of AAS.

\section{ACKNOWLEDGMENT}

This work has been conducted in the framework of the IMAGE $5 \mathrm{G}$ project. The authors would like to acknowledge IMAGE $5 \mathrm{G}$ research project consortium that consists of Centria University of Applied Sciences, Nokia, PehuTec, Pohjonen Group, Siipotec, Finnish Communication Regulatory Authority, Finnish Meteorological Institute and Tekes - Finnish Funding Agency for Innovation and CORE++ project consortium.

\section{REFERENCES}

[1] Nokia Networks-Active Antenna Systems: A step-change in base station site performance, Oct. 2013, White Paper. Available: http://resources.alcatel-lucent.com/asset/200016.

[2] J. Erkkilä et al, "Antenna configuration comparison in challenging NLOS locations," in European Conference on Networks and Communications, Oulu, Finland, 2017, to be published.

[3] J. T. J. Penttinen, "Planning of the LTE-advanced radio network," in The LTE-Advanced Deployment Handbook: The Planning Guidelines for the Fourth Generation Networks, J. T. J. Penttinen, Ed. ohn Wiley \& Sons, Ltd., January 2016, pp. 257-292.

[4] Effects of building materials and structures on radiowave propagation above about $100 \mathrm{MHz}$," ITU-R P. 2040-1, 07/2015.

[5] R. Rudd et al, "Building materials and propagation final report, ofcom".

[6] D. O. Reudink and M. F. Wazowicz, "Some Propagation Experiments Relating Foliage Loss And Diffraction Loss at X-Band and UHF Frequencies," IEEE Trans. Commun., vol. 21, pp. 1198-1206, 1973.

[7] N. Savage et al, "Radio wave propagation through vegetation: Factors influencing signal attenuation," Radio Sci., vol. 38, 2003.

[8] Attenuation in vegetation, ITU-R P. 833-9, 09/2016.

[9] Propagation data and prediction methods required for the design of terrestrial line-of-sight systems, ITU-R P. 530-16, 07/2015, .

[10] T. Oguchi, "Electromagnetic Wave Propagation and Scattering in Rain and Other Hydrometeors," Proc IEEE, vol. 71, pp. 1029-1078, 1983.

[11] J. A. Morrison, M. J. Cross and T. S. Chu, "Rain-Induced Differential Attenuation and Differential Phase Shift at Microwave Frequencies," Bell Syst. Tech. J., vol. 52, pp. 599-604, 1973.

[12] CCIR, "Rep. 721-2 attenuation by hydrometeors, in particular precipitation, and other atmospheric particles, vol V." XVII Plenary Assembly, Dussendorf, 1990.

[13] The CORE++ project. Available: http://core.willab.fi/.

[14] "Huawei E392 LTE USB Stick, Datasheet." 2011-5-03.

[15] 2J s.r.o. "2J300M (datasheet)". Available: http://www.2jantennae.com/images/products/2J300M.pdf.

[16] Optimization Solution Sheet. Available: http://www.anite.com/sites/default/files/1Optimization.pdf 